\title{
Assessing stress in surgery: using validated tools to improve patient safety
}

\author{
A.-S. Alexopoulos $\cdot$ O. Shariq
}

Received: 18 October 2010/Accepted: 2 November 2010/Published online: 18 November 2010

(C) Royal Academy of Medicine in Ireland 2010

\section{Dear Editor,}

It is becoming increasingly apparent that the technical proficiency of a surgeon is only one of many factors that influence surgical outcomes. Boyle et al. [1] have highlighted what has been termed a 'systems approach' which acknowledges the contribution of non-technical skills, such as stress-coping ability, to safe surgical practice. Unlike many other high-risk industries, such as aviation and the military, there is currently no stress/crisis management training to safeguard surgeons against the detrimental effects of excessive stress [2]. It is, therefore, important to address this issue through the use of consistent measures to identify and assess the stress during surgery.

Interestingly, through the use of a general self-efficacy (GSE) questionnaire, Boyle et al. [1] found a lack of correlation between trainees' GSE scores and surgical performance on laparoscopic and bench model-type stations. Whilst this demonstrates the inherent difficulty in measuring non-technical skills, it also emphasises the need for a reliable and accurate tool for assessing the overall effect of stress on surgical performance. Arora et al. [3] have recently proposed the imperial stress assessment tool (ISAT) which is a three-component stress measure consisting of salivary cortisol, heart rate and self-reported stress levels. It was demonstrated that both objective markers correlated well with self-reported stress, with high specificity and sensitivity. Notably, ISAT measures stress not only before, but also during the operative procedure. This

\footnotetext{
A.-S. Alexopoulos $(\bowtie) \cdot$ O. Shariq Imperial College London, London, UK

e-mail: aa3405@imperial.ac.uk

O. Shariq

e-mail: omair.shariq@doctors.org.uk
}

overcomes the limitations inherent in self-reporting questionnaires which rely heavily upon the student's ability to reliably assess their own stress levels prior to the surgery.

Importantly, the ISAT is a non-intrusive method, taking no more than $10 \mathrm{~min}$ of the surgeon's time. This is integral to the implementation of such a measure, as it renders it feasible for use within the busy operating theatre setting. Its practicality and validity make ISAT a good tool for assessing stress in surgery, allowing for detailed feedback to be given to surgeons, and facilitating targeted training in stress management. Not only will further factual evidence encourage the surgical community to acknowledge stress as a relevant issue, but it will also help formulate simulation-based training programs that closely mimic real-life experiences. The use of ISAT alongside validated measures of other non-technical (e.g. decision-making and leadership) and technical skills will be fundamental to the application of a 'systems approach' to such programs. The adoption of a holistic strategy to improving the skills required during surgery will enhance the surgical performance and ultimately lead to improved patient care.

\section{References}

1. Boyle E, Kennedy AM, Doherty E, O'Keeffe D, Traynor O (2010) Coping with stress in surgery: the difficulty of measuring nontechnical skills. Ir J Med Sci. doi: 10.1007/s11845-010-0578

2. Arora S, Sevdalis N, Nestel D, Tierney T, Woloshynowych M, Kneebone R (2009) Managing intraoperative stress: what surgeons want from a crisis training program? Am J Surg 197:537-543. doi: 10.1016/j.amjsurg.2008.02.009

3. Arora S, Tierney T, Sevdalis N et al (2010) The imperial stress assessment tool (ISAT): a feasible, reliable and valid approach to measuring stress in the operating room. World J Surg 34: 1756-1763. doi:10.1007/s00268-010-0559-4 